\title{
Fourth Annual Report of the Prairie Nest Records Scheme
}

\author{
by Elmer L. Fox, Regina
}

The Prairie Nest Records Scheme has completed its fourth year of operation. The number of cards received this year was somewhat smaller, but excellent records were submitted on many of these. There were fewer records of breeding waterfowl, presumably owing to the drought conditions prevailing throughout the Prairie Provinces. By January $15,1962,37$ contributors had submitted 814 cards for 135 species.

A summary of the first four years of the Scheme's operation is being prepared, and this mimeographed report will be sent to all contributors. A limited number of copies will be available for others who write for them to the Prairie Nest Records Scheme.

Similar nest record schemes are in operation in the Maritimes, in Ontario, and in British Columbia. There is no coverage for Quebec. The territory covered by the Saskatchewan Natural History Society's Prairie Nest Records Scheme includes the Prairie Provinces and the Northwest Territories.

A new supply of cards for 1962 will be sent out to all 1961 contributors. New contributors who have nesting information to submit should request cards. For new contributors, we should like to point out again that information is required on even the common birds breeding in your area, and that as much information as possible should be recorded on each nest found. A card recording observations from the time the eggs are laid until the young leave the nest is more valuable than a single observation. We urge you, too, to disturb as little as possible the birds you are studying.

Send requests for record cards or for the report on the Scheme's operation to: The Prairie Nest Records Scheme, Saskatchewan Natural History Society, Saskatchewan Museum of Natural History, Regina.

CONTRIBUTORS: W. Anaka, Genevieve Belliveau, F. Brazier, D. Buckle, H. Burns, J. R. Caldwell, B. Capusten, D. Chandler, $H$.
Copland (with W. Johnston, H. Hasford, G. Smith), R. Derkutch, E. A. Dodd, E. Fox (with $R$. Fox), G. Gletcher, Joyce Gunn, $\dot{H}$. Hasford, D. Hatch, H. Hedger, R. Klimach, E. Kuyt, L. $V$. and V. B. Latta, J. Lane, R. McCall, Mamie McCowan, S. A. Mann, S. Manu (with J. Bennett), W. J. Merilees, Daisy Myers, R. W. Nero (with R. Carson), K. D. Patton, J. M. Provick, Barbara Robinson, R. Sanderson (with W. Fleming), V. Schmidt, G. Smith, L. Tohr, E. J. White, S. Zazelenchuk.

SPECIES RECORDED: Common Loon (1), Horned Grebe (5), Eared Grebe (8), Piedbilled Grebe (8), Double-breasted Cormorant (4), American Bittern (1), Whistling Swan (2), Canada Goose (6), White-fronted Goose (3), Mallard (21), Pintail (6), Green-winged Teal (6), Blue-winged Teal (2), American Widgeon (3), Shoveler (5), Redhead (2), Canvasback (5), Lesser Scaup (2), Ruddy Duck (5), Red-breasted Merganser (1). Sharp-shinned Hawk (1), Cooper's Hawk (4), Red-tailed Hawk (6), Swainson's Hawk (3), Rough-legged Hawk (1), Ferruginous Hawk (1), Bald Eagle (3), Marsh Hawk (4), Gyrfalcon (1), Pigeon Hawk (1). Sparrow Hawk (2), Spruce Grouse (1), Ruffed Grouse (1), Willow Ptarmigan (3), Sharp-tailed Grouse (5), Gray Partridge (2), Virginia Rail (2), Sora (2) American Coot (23), Piping Plover (2), Killdeer (22), Common Snipe (1), Upland Plover (2), Spotted Sandpiper (2), Willet (4), Least Sandpiper (1), Marbled Godwit (2), American Avocet (5), Wilson's Phalarope (8), Long-tailed'Jaeger (1), Herring Gull (1), Franklin's Gull (1), Common Tern (6), Arctic Tern (1), Black Tern (4), Rock Dove (2), Mourning Dove (15), Blackbilled Cuckoo (17), Screech Owl (1), Great Horned Owl (7), Hawk Owl (2), Burrowing Owl (1.), Long-eared Owl (1), Short-eared Owl (4), Ruby-throated Hummingbird (1), Yellowshafted Flicker (5), Red-headed Woodpecker (1), Downy Woodpecker (3), Eastern Kingbird (14), Western Kingbird (6), Eastern Phoebe (24), Traill's Flycatcher (1), Least Flycatcher (5), Dusky Flycatcher (5), Western Wood Pewee (1), Horned Lark (9), Tree Swallow (13), Bank Swallow (6), Rough-winged Swallow (1), Barn Swallow (46), Cliff Swallow (6), Blue Jay (2), Black-billed Magpie (12), Common Raven (1). Common Crow (19), Black-capped Chickadee (3), House Wren (27), Long-billed Marsh Wren (5), Short-billed Marsh Wren (4), Eatbird (10), Brown Thrasher (8), Robin (43), Hermit Thrush (1), Swainson's Thrush (1), Gray-cheeked Thrush (3), Veery (2), Mountain Bluebird (7), Sprague's Pipit (1), Cedar Waxwing (11), Loggerhead Shrike (2), Starling (8), Red-eyed Vireo (4), Warbling Vireo (6), Yellow Warbler (13); Palm Warbler (1), Ovenbird (1), Yellowthroat (2), House Sparrow (4), Bobolink (3). Western Meadowlark (10), Yellow-headed Blackbird (29), Redwinged Blackbird (42), Baltimore Oriole (3), Brewer's Blackbird (13), Common Grackle (7), Western Tanager (1), Rose-breasted Grosbeak (1), Indigo Bunting (1), Purple Finch (1), Hoary Redpoll (1), Pine Siskin (3), American Goldfinch (2), Lark Bunting (1), Savannah Sparrow (14), Báird's Sparrow (1), Vesper Sparrow (19), Lark Sparrow (2), Slatecolored Junco (3), Tree Sparrow (8), Chipping Sparrow (6), Clay-colored' Sparrow (13), Harris' Sparrow (3), White-crowned Sparrow (2), Song Sparrow (1i), Chestnut-collared longspur (6). 


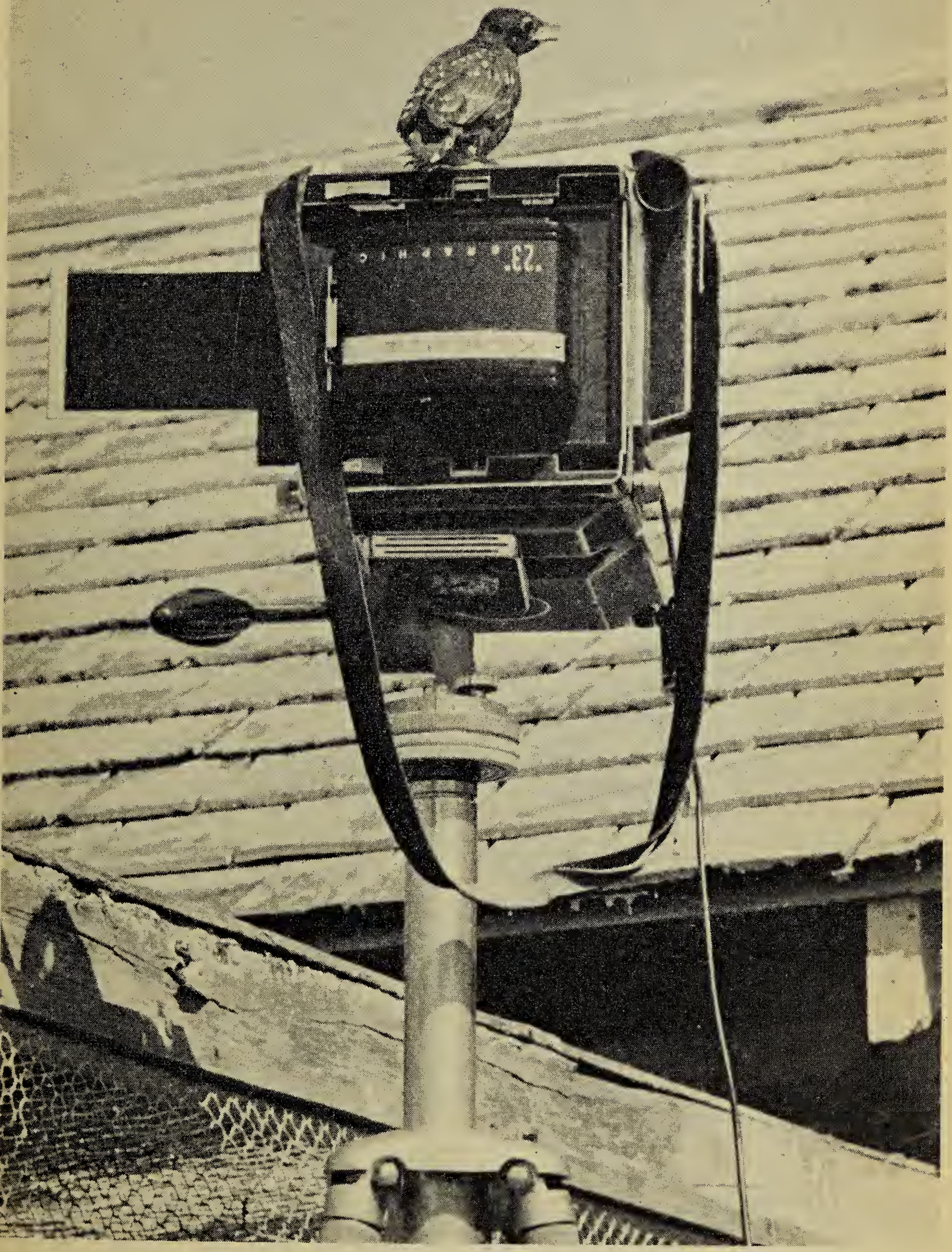

